\title{
Lightframe On-sight Key species Investigation (LOKI)
}

\author{
The art of imaging minute plankton species on-the-fly
}

\author{
Jan Schulz ${ }^{1,4}$, Kristina Barz ${ }^{1}$, Dirk Mengedoht ${ }^{1}$, Thomas Hanken ${ }^{2}$, Heiko Lilienthal ${ }^{2}$, Norbert Rieper ${ }^{2}$, Jan Hoops ${ }^{2}$, \\ Kurt Vogel $^{3}$, Hans-Jürgen Hirche ${ }^{1}$ \\ ${ }^{1}$ Alfred Wegener Institute \\ Am alten Hafen 26 \\ 27568 Bremerhaven \\ www.awi.de \\ ${ }^{2}$ iSiTEC GmbH \\ Stresemannstraße 46 \\ 27570 Bremerhaven \\ www.isitec.de \\ ${ }^{3}$ MedeaAV Multimedia $\mathrm{GmbH}$ \\ Am Weichselgarten 23 \\ 91058 Erlangen \\ www.medea-av.de \\ ${ }^{4}$ IMARE-Institute for Marine Resources \\ Klußmannstraße 1 \\ 27570 Bremerhaven \\ www.imare.de
}

\begin{abstract}
In this short note we give an overview on the development of a new versatile plankton video recording system named LOKI. In addition to the introduction of technical specifications and background information of the device we also demonstrate a possible scientific application in a case study performed in an upwelling area off the Namibian coast.
\end{abstract}

\section{INTRODUCTION}

Plankton forms the foundation of the food chain in limnic and marine waters and its availability is fundamental to ecosystem dynamics. Rapid mapping of plankton abundance in combination with taxonomic and size composition is very important for aquatic environmental research, but difficult or impossible to accomplish using traditional techniques. Common sampling strategies, like towed nets, have methodborne shortcomings like low spatial resolution, intrinsic depth integration and time consuming sample analysis. Thus, their use is limited for studying distribution patterns on adequate scales in the vicinity of hydrographic fronts and clines, although such structures determine to a high degree community assemblages and trophic interactions.

Since the 1950s optical systems have been developed to overcome these limitations (e.g. [1], [2]) and today digital technology allows in-situ imaging combined with data from a complex network of environmental sensors. The optical systems image objects dispersed in water, while sensors record a multitude of associated hydrographic parameters. Two major approaches can be distinguished that provide a number of advantages and aid net-based investigations:

1. Systems equipped with line sensors acquire object information in consecutive rows, similar to light barrier systems. The use of highly collimated light over short distances reduces scatter and high background noise from stray light. Modern systems, like contemporary Laser-OpticalPlankton-Counters, give information on cross sections or composited contour lines [3]. High quality shadow images can be captured by using line scan cameras across flow-through channels [4]. By gauging flow these systems also allow quantification of scanned volumes and to composite consecutive row data to $2 \mathrm{D}$ shadow images.
2. Imaging with cameras is a more ambitious and data intensive approach, which is faced with two main challenges. The first is taking images of objects with sufficient quality for taxonomic identification. Therefore, a relatively short distance between camera and object is required to reduce object blurring at high particle densities and reduced water clarity [5]. Covering size ranges from 0.1 to $10 \mathrm{~mm}$ with a high depth of field (DOF) is close to the feasible border of optical laws. High magnifications at short distances result in a small DOF, and thus, in a high percentage of out-of-focus objects [6]. Furthermore, increased magnification results in a higher relative translational displacement of the drifting fluid. This requires short shutter times $<1 \mathrm{~ms}$ to avoid motion blurring [7]. The second difficulty is the quantification of objects, which requires the precise estimation of the volumes imaged per frame. Several systems estimate volumes of acquired images by using software and calculate DOF by the sharpness of the imaged particles [8]. Through-flow chambers allow physical limitation of the volume to the DOF and easily manageable and reproducible illumination. However, finescale patterns and fragile species are affected by the concentration process for the chamber [9]. To display surface and internal structures of small and transparent organisms, dark field illumination has proven to be an excellent choice [10] [11]. Several systems profile plankton and marine snow in volumes between 500 to $6500 \mathrm{ml}$ (e.g. [12], [11], [13]). The high volumes scanned by such devices often suffer from giving low detailed resolution for taxonomic identification of small organisms, like copepods.

Here we present a new approach in the development of an in-situ plankton imaging system, called Lightframe On-sight Key species Investigation (LOKI). It allows to image minute objects of sizes below $100 \mu \mathrm{m}$ with high resolution. Modular components allow the use as devices towed from research vessels, as moorings on unmanned platforms, as well as bench top use. This paper describes the LOKI device and presents first results from a research cruise off the Namibian coast. 


\section{MATERIAL \& METHODS}

In the design of the Lightframe On-sight Key species Investigation (LOKI) system (Figure 1a) five major units can be distinguished. These units operate as independent functional groups that capture or process data and are interconnected via internal LAN.

\section{A. Imaging unit}

For different applications, like towed operation or moorings, three types of imaging units were developed. The approaches presented here are based on illumination techniques that either project a light frame of high luminous flux into the water or constrain the volume physically with transparent boundaries. High magnifications at short distances result in a short depth-of-field (DOF). High f-values (small aperture opening) enlarge DOF by minimising the circles of confusion, but reduce light available during exposure. To
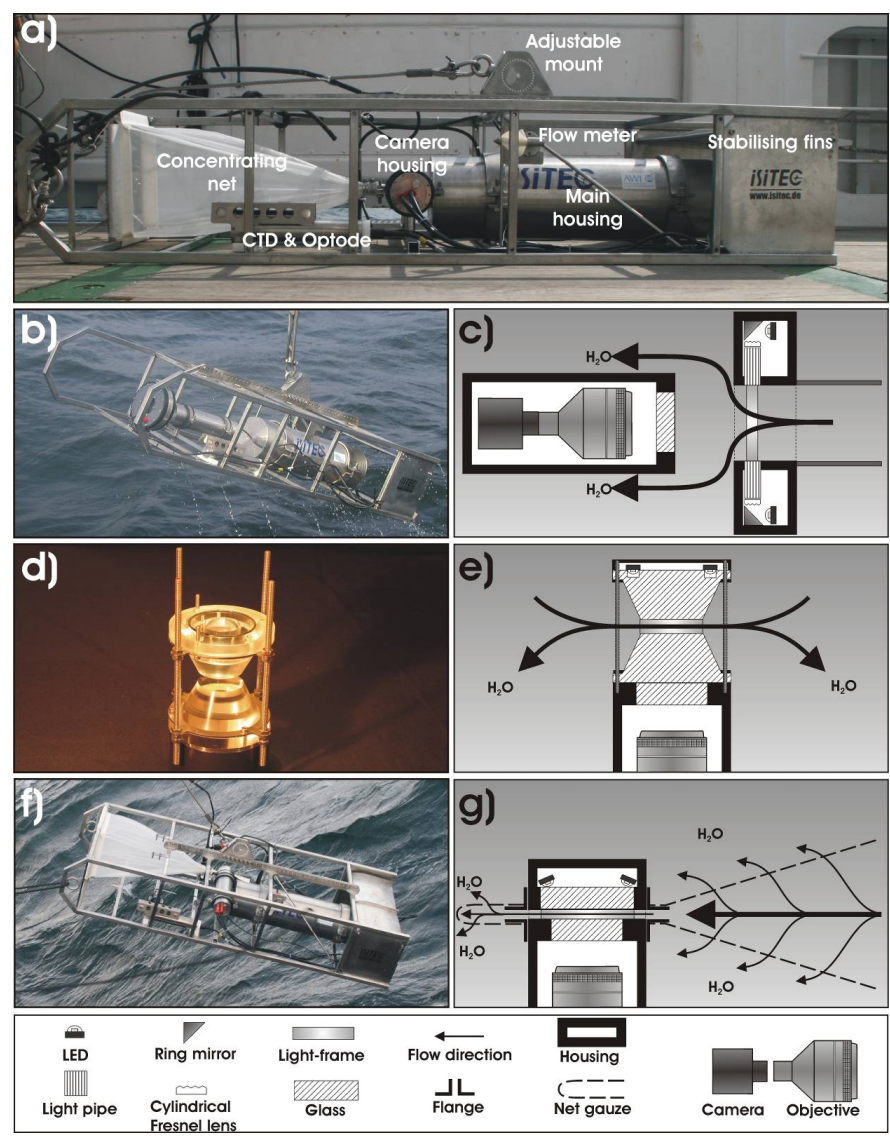

Figure 1: Overview and set-ups of the LOKI device for different applications. a) Overview of the main parts. b) Light-ring application for free sample through-flow. In the ring centre a disc of collimated light illuminates particles from all sides simultaneously to avoid casting shadows (Schulz 2007). c) Schematic view of the light ring setup. d) The imaging unit for moored applications during maintenance. e) Schematic view of the image head for moorings. f) Imaging unit equipped with a net for concentrating samples, through-flow chamber and cod end for additional sample collecting. g) Schematic view of the combined net and imaging unit. quantify the volume scanned per frame requires knowledge of width, height and depth of the observed volume. While the first two are given by the dimensions of the photosensitive sensor, the infinite principal axis can be optically constrained by illumination in the narrow DOF.

Particles within this area are illuminated. Only directly illuminated objects are visible for the camera, Objects out of the focus range are nearly invisible. Under physically constraint conditions objects can not appear in a certain distance before and behind the observation volume's depth, as it is equipped by transparent materials. This produces clearer images, as no fuzzy background noise may appear. Relative water movement replaces the water and entrained objects between two frames. This movement may be caused by towed operation, tidal currents or mechanically induced flow.

The camera aims with an angle of $90^{\circ}$ at the light frame or physically constrained illuminated observation volume (Figure $1 \mathrm{c}, \mathrm{e}, \mathrm{g})$. With a frequency of up to $15 \mathrm{fps}$ a digital camera with four million pixels (Prosilica GE2040) takes images of objects within the light frame with shutter times below $150 \mu \mathrm{s}$. To allow short shutter times, the development of illumination devices with high light flux and precise targeting is a pivotal precondition for in-situ imaging of small planktonic specimens. As light sources all units use high power Light Emitting Diodes (LED's). LED's are operated with an approximately tenfold higher forward current than specified. Flash mode ensures that integral of energy over time is similar as in continuous mode and assures health status of LED's.

To account for different questions a variety of imaging units were designed (Figure 1b-g). They cover the entire application range for detection of objects from low to high densities, for use on towed platforms aboard of research vessels, on moorings and in the laboratory. Units are operated with 1.3 to 4 megapixel GigE cameras. Image acquisition runs with $15 \mathrm{fps}$ at full resolution. Frames trigger a high power LED flash unit, which allows exposure times $<100 \mu$ s.

\section{B. Multi Sensor Carrier Network System}

Environmental information from arbitrary sensors are gathered by a newly developed versatile Multi-Sensor-CarrierSystem (MUSECS, Figure 2). Its task is to support the main unit by supplying environmental data on demand in a specified format. MUSECS is designed to attach any desired customary sensor automatically to the system via universal interface. Each attached sensor is controlled by a microcontroller for protocol adaptation and signal conversions, if required. Connection and power supply is managed via industrial CAN-Bus. A CTD, an oxygen optode and a speed meter are continuously attached. The system is prepared to include further sensors, like fluorometers or (Laser-) Optical Plankton Counters. Communication between MUSECS and the main processing unit, where data are stored, takes place over an independent 100MBit Ethernet network. 


\section{Main processing unit}

The main processing unit operates a Windows XP on an Intel Dual Xeon board with $2.8 \mathrm{GHz}$ clock rate and $2 \mathrm{~GB}$ main memory. Environmental parameters are polled from MUSECS and logged in intervals of one second. The camera is connected by Gigabit Ethernet. The recording software preprocesses the data stream of approximately $60 \mathrm{MB} \mathrm{s}^{-1}$ in real time and logs frame rate. Only segments within each image frame that contain objects are stored as Area Of Interest (AOI)

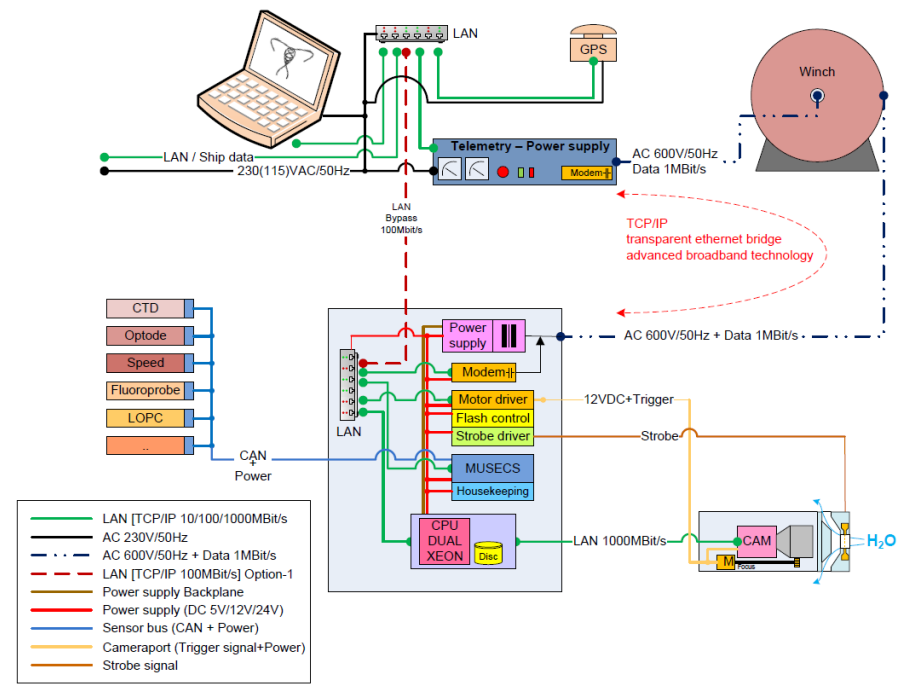

to reduce required storage space. Minimum object size for

Figure 2: Components and schematic wiring of the LOKI system.

detection can be manually adjusted. Stored images carry time stamps to allow correlation with environmental parameters of the respective time frame.

\section{Surface communication}

The communication between surface and underwater unit is achieved by a transparent TCP/IP Ethernet bridge (Figure 2). The used multi-frequency modem modulates the signal onto the power supply voltage of 600VAC and enables the use on ships with hawsers bearing just two-conductor cables for connection. Ideal conditions allow communication with a maximum speed of $1 \mathrm{MBit} \mathrm{s}^{-1}$ over more than $8 \mathrm{~km}$ of copper coax cable. Surface data, like e.g. GPS, are transferred to the underwater unit and included in the log files. A VPN tunnel can be established to monitor operation online. The use of TCP/IP standards also allows remote operation and data access via existing inter- or intranet connections. Thus, the device can be operated on unmanned stations.

\section{E. Decksystem}

Via VPN operation can be continuously observed and first impressions about particle densities from the camera received on-line. After operation data storage and handling is managed by a SQL database backend. This easily allows formulation of queries to investigate relationships between recorded objects and hydrographic information. Modules for manual and automatic classification further ease processes of initial classification. Development of the software package LOKIBrowser allows making queries about images related with physical parameters and other entities. Furthermore LOKIBrowser calculates characteristic parameters of each image that can be used for automated image characterisation. Statistics and chartings are performed with the open source software suite R-Project for statistical computing (www.Rproject.org). Internationally stipulated sustainable data storage is achieved by an optional import option for the World-DataCentre MARE database PANGAEA.

\section{F. Data}

Data presented here were sampled by the LOKI system off the Namibian coast $\left(-19.924^{\circ} \mathrm{N}, 011.217^{\circ} \mathrm{E}, 29^{\text {th }}\right.$ March 2008 , 16:26 UTC) during a cruise aboard the German R/V Maria S. Merian. A vertical haul (heave speed $\sim 0.5 \mathrm{~m}^{*} \mathrm{~s}^{-1}$ ) from $400 \mathrm{~m}$ depth to surface was carried out with the net-equipped imaging unit $(50 \mu \mathrm{m}$ gauze, Figure $1 \mathrm{f})$. Primary data are published within the data base PANGAEA of the World-DataCentre Mare and can be freely accessed:

\section{doi:10.1594/PANGAEA.708309 (Physical oceanography) doi:10.1594/PANGAEA.708195 (Images)}

Images of dominant plankton groups were classified in the LOKI-Browser. A data subset was compiled, containing specimens of the dominant copepod families: Aetideidae, Calanidae, Metridinidae, Oncaeidae and Rhincalanidae. Classified objects were assigned to their connected to their respective environmental parameters: Depth, Temperature, Salinity and Oxygen concentration. These parameters were charted against each other as pair plots. Instead of continuous lines dots indicate the individual position of a specimen. A colour code was introduced to visualise differences between different families.

\section{RESULTS}

The LOKI system provides clear images of plankton species exposed on-the-fly with the concentrating net unit. The high power LED flash unit allow imaging at shutter times of $100 \mu \mathrm{s}$. No motion blurring is observed while particles pass the cameras field of view in the through-flow chamber. Images are sufficient to be determined by specialists to at least family level. In many cases identification to genus or even species level is possible. In seldom cases specimens are imaged in an angle that disallows identification. Data gathered by the MUSECS network system allows assigning physical parameters in the vicinity of an imaged object on scales $\leq 50 \mathrm{~cm}$.

Copepods were found to be unevenly distributed in the water column and showed distinct vertical patterns (Figure 3). In the upper part of the water column specimen density was highest close to the surface. A second peak was observed 

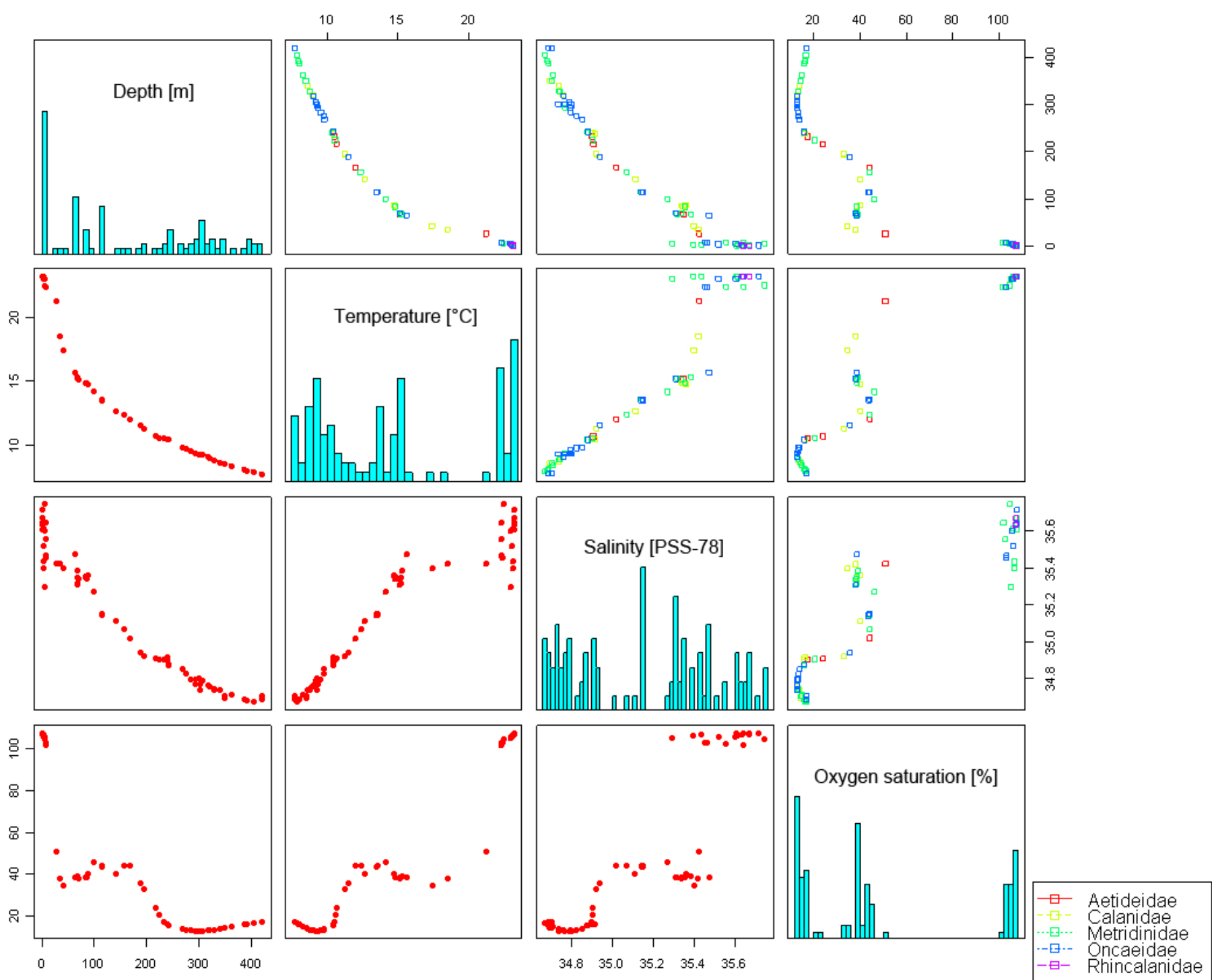

Figure 3: Distribution of dominant copepod families in relation to hydrographic parameters in their direct vicinity. Lower left charts show distribution of all included copepods, while the upper right charts are colour coded according to the family the respective copepod belonged to.family. The histogram on the diagonal shows the overall copepod distribution in relation to the physical parameters.

around $80-120 \mathrm{~m}$ depth and a third accumulation was found in the range between 260 and $340 \mathrm{~m}$ depth, where lowest oxygen concentrations were recorded.

Specimens of the family Aetideidae were only observed below the thermocline and above the depth of the oxygen minimum zone. Aetideidae were not observed below the oxygen minimum zone. Within the oxygen minimum zone specimens of the family Oncaeidae were most abundant. On the lower side of this depletion zone number of specimens of the familiy Metridinidae exceeded others. A clear separation was seen between Metridinidae and Oncaeidae around the oxygen minimum zone. While Oncaeidae concentrated at lowest oxygen levels, Metridinidae specimens flanked this

aggregation from the lower side. Specimens of the family Similar to the Aetideidae the Calanidae were found between the oxygen minimum zone and the thermocline and were mainly detected in the vicinity of small scale gradients. Few specimens of the family Rhincalanidae were detected close to the surface.

\section{DISCUSSION}

Plankton is assumed to occur in micropatches $(<10 \mathrm{~m})$ that have large impact on trophodynamic interactions in marine systems [14]. Thus, the required scale for sampling is finer than can be practically achieved by most sampling devices. Multi-open-close nets improve sampling, but are still prone to movements of the research vessel and waves making it impossible to assign a specimen to an exact position, depth or physical parameter. It can just be said that specimens are abundant within the depth range covered by the net. Data presented here demonstrate that dominant copepod families 
are unevenly distributed in certain depths and that a spatial sample resolution of a few meters is desirable. Specimens of the family Oncaeidae dwell to a large extend in the oxygen minimum zone (OMZ). Active migration into $\mathrm{OMZ}$ and adaptation to low oxygen levels is known for several copepods [15]. Although nothing can be said about diurnal vertical migrations from a single haul, it can be supposed that this layer may offer reduced predation pressure. In combination with reduced light conditions in these depths the reflective and optically dense Oncaeidae specimens are probably less accessible for predators. The biological background for the observed separation between Oncaeidae and Metridinidae in the depth of the OMZ remains unclear. Close to the surface wind shearing causes higher fraction and insertion of air bubbles. Tiny specimens can stick to bubbles, when getting in contact. Here, Rhincalanidae specimens benefit from their relatively large size and can utilise the high primary production close to the surface. Observed inhabitation preferences of specific water masses by copepod specimens of the families Aetideidae and Calanidae is congruent with findings by other authors [15], although knowledge about appearance close to hydrographic gradients is limited.

The advantage provided by LOKI in-situ imaging is the high sampling resolution on the centimetre scale. It allows investigating finer scale patterns, impossible to resolve with classic approaches. Method-borne integration of nets deletes such information, although important for the understanding of marine ecosystems. Additionally, the assignment of environmental parameters in the direct vicinity of a specimen or object enhances options for numerical approaches in plankton ecology. Still increasing progress in camera and lightning techniques allow gaining insight into biological processes that were so far not resolvable in such quality and scales [cf. 16]. Sophisticated image recognition software under development will aid manual classification in future. In addition to standard parameters, like Hu-moments, Fourierdescriptors or texture analysis, it includes new form based feature extractions [17] [18].

Like all video plankton recorders the LOKI system images objects within a small band of constraint diameter in a water mass with relative displacement. Only objects within this band can be detected. As the observed volume is rather small, low abundant species have a higher probability to remain undetected [14] [19]. Thus, the word key was included in Lightframe On-sight Key species Illumination (LOKI).

\section{ACKNOWLEDGEMENT}

Technical developments were funded by the Bremerhavener Gesellschaft für Investitionsförderung und Stadtentwicklung $\mathrm{mbH}$ (BIS 56008/2-Z and BIS 68036/2-Z), co-financed by the European Community.

\section{REFERENCES}

[1] K.G. Foote (2000) Optical methods. In Harris RP, Wiebe PH, Lenz J, Skjoldal HR, Huntley M (eds). ICES zooplankton methodology manual, pp 259-295.

[2] P.H. Wiebe, M.C. Benfield (2003) From the Hensen net toward fourdimensional biological oceanography. Prog Oceanogr 56:7-136

[3] A.W. Herman, B. Beanlands and E.F. Phillips. 2004. The next generation of Optical Plankton Counter: The Laser-OPC. J Plank Res 26: 11351145 .

[4] S. Samson, T. Hopkins, A. Remsen, L. Langebrake, T. Sutten J. Patten (2001) A system for high-resolution zooplankton imaging. IEEE J Ocean Eng 26:671-676.

[5] J. Pollio, R. Meyer, L.D. Sivak (1979) Model analysis of underwater photographic and visibility systems from observed data. In: Proc. Soc. Photo-Optic Instr. Engin., Ocean Optics 6, 208: 232-241.

[6] J.R. Strickler (1977) Observation of swimming performances of planktonic copepods. Limnol Oceanogr 22:165-170.

[7] A.T. Mustard, M.D. Conquer, J.T. Allen (2003) Laboratory evaluation of a high specification digital CMOS camera for imaging zooplankton at high towing speeds. Southampton Oceanography Centre, Internal Document No. 89, pp 17.

[8] C.S. Davis, S.M. Gallager, M. Marra, W.K. Stuart (1996) Rapid visualization of plankton abundance and taxonomic composition using the video plankton recorder. Deep Sea Res 43:1947-1970.

[9] K. Wieland, B. Hermann, J. Kreikemeier, J. Lenz, S. Mees, D. Schnack (1992) Preliminary results of the small-scale distribution of herring larvae in the English Channel as obtained by means of a towed video recording system. Deep Sea Res 30:331-342.

[10] U. Kils (1989) On the micro-structure of micro layers, Results of an insitu zooplankton-counter. ICES CM 1989/L:15.

[11] G. Gorsky, M. Picheral, L. Stemmann (2000) Use of the underwater video profiler for the study of aggregate dynamics in the North Mediterranean. Estuar Coast Shelf Sci 50:121-128.

[12] M.C. Benfield, R.F. Shaw, C. Schwehm (2000) Development of a vertically profiling, high-resolution, digital still camera system. ONR Ann Rep Office Nav Res, NR Grant No.: N00014-98-1-0563, pp 6.

[13] M. Lunven, P. Gentien, K. Kononen, E. Le Gall, M.M. Daniélou (2003) In situ video and diffraction analysis of marine particles. Estuar Coast Shelf Sci 57:1127-1137.

[14] C.S. Davis, S.M. Gallager, A.R. Solow (1992) Microaggregations of Oceanic Plankton Observed by Towed Video Microscopy. Science 257:230-232.

[15] N. Loick, W. Ekau, H.M. Verheye (2005) Water-body preferences of dominant calanoid copepod species in the Angola-Benguela frontal zone. Afr J Mar Sci 27(3):597-608.

[16] P.H. Wiebe, M.C. Benfield (2003) From the Hansen net towards fourdimensional biological oceanography. Prog Oceanogr 56:7-136.

[17] L.J. Latecki, R. Lakämper (2000) Shape similarity measure based on correspondence of visual parts. IEEE Trans. Pattern Analysis and Machine Vision. 22(10):1-6.

[18] ISO/IEC TR 15938-8 (2002) Information technology - Multimedia content description interface - Part 8: Extraction and use of MPEG-7 descriptions.

[19] M.C. Benfield, C.S. Davis, P.H. Wiebe, S.M. Gallager, R.G. Lough, N.J. Copley (1996) Video plankton recorder estimates of copepod, pteropod and larvacean distributions from a stratified region of Georges bank with comparative measurements from a MOCNESS sampler. Deep-Sea Res II, 43:1925-1946. 